\title{
BMJ Open Focused ultrasound examination of the chest on patients admitted with acute signs of respiratory problems: a study protocol for a pragmatic randomised controlled multicentre trial
}

\author{
M Riishede, ${ }^{1,2,3}$ C B Laursen, ${ }^{2,4}$ L S Teglbjærg, ${ }^{3}$ A T Lassen, ${ }^{2,5}$ G Baatrup ${ }^{1,2}$
}

To cite: Riishede M, Laursen CB, Teglbjærg LS, et al. Focused ultrasound examination of the chest on patients admitted with acute signs of respiratory problems: a study protocol for a pragmatic randomised controlled multicentre trial. BMJ Open 2016;6:e012367. doi:10.1136/bmjopen-2016012367

- Prepublication history and additional material is available. To view please visit the journal (http://dx.doi.org/ 10.1136/bmjopen-2016012367).

Received 20 April 2016 Revised 4 July 2016 Accepted 28 July 2016

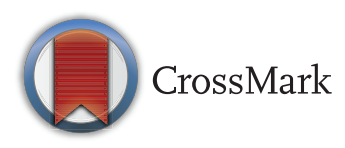

For numbered affiliations see end of article.

Correspondence to Minna Riishede; Minna. Riishede@rsyd.dk

\section{ABSTRACT}

Introduction: Patients with acute respiratory problems poses a diagnostic challenge because similar symptoms can be caused by various pathological conditions. Focused ultrasound examination (f-US) of the heart and lungs has proven to increase the diagnostic accuracy in these patients. In this protocol of a randomised multicentre trial, we study the effect of $f$-US of the heart and lungs in patients with respiratory problems performed by emergency physicians (EP) as soon as the patient arrives to the emergency department (ED). The primary outcome is the number of patients with a correct presumptive diagnosis at 4 hours from admission.

Methods and analysis: This is a semiblinded randomised prospective study. 288 patients will be included and randomised into the control or intervention group. All patients receive a standard diagnostic evaluation by the EP to assess the primary presumptive diagnosis. Investigators are EP, with varying degrees of experience in $\mathrm{f}$-US, who perform an $\mathrm{f}$-US of the heart and lungs in patients in both treatment arms. f-US results in the intervention group are non-blinded to the treating EP to be included in the assessment of the 4-hour presumptive diagnosis. As standard for correct diagnosis, we perform a blinded journal audit after discharge. As primary analysis, we use the intention-to-treat analysis.

Conclusions: This study is the first multicentre trial in EDs to investigate whether f-US, in the hands of the $E P$, increases the proportion of correct diagnosis at 4 hours after arrival when performed on patients with respiratory problems.

Ethics and dissemination: This trial is conducted in accordance with the Helsinki II Declaration and approved by the Danish Data Protection Agency and the Committee on Biomedical Research Ethics for the Region of Southern Denmark. Results will be published in accordance with the CONSORT statement in a peer-reviewed scientific journal regardless of the outcome.

Trial registration number: NCT02550184; Pre-results.

\section{INTRODUCTION}

Patients arriving to the emergency department (ED) with symptoms of acute respiratory problems are often a diagnostic challenge. Despite similar clinical symptoms, the aetiology can vary and require different treatments and interventions. ${ }^{1}$ Earlier studies have found that at least one diagnosis was missed in $>20 \%$ of these patients and that $>30 \%$ of them received a suboptimal treatment leading to increased length of stay at hospital and increased mortality. ${ }^{1-3}$ The assessment of a fast and correct diagnosis is therefore of high importance in these patients where instant treatment may be lifesaving. ${ }^{124-6}$

Focused ultrasound examination (f-US) of the lungs and heart has, over the past years, emerged as a fast, non-invasive, bedside tool for diagnosing diseases in the chest. This has led to the development of evidence-based guidelines describing indications, technical performance and interpretation of ultrasound of the lungs and heart, ${ }^{7-9}$ as well as guidelines for the implementation of f-US on patients in the EDs and intensive care units. $^{10-13}$

Alongside standard paraclinical (eg, blood samples, ECG, chest X-ray) and physical examination, several trials have investigated the impact of an f-US on the diagnostic accuracy of some of the most common diseases encountered in patients with symptoms of respiratory problems in the EDs (eg, pneumonia, chronic obstructive lung disease, pulmonary oedema, lung embolism, acute heart failure). ${ }^{2} 3$ 14-29 Most of these studies proved a high diagnostic accuracy when using an f-US alongside standard diagnostic testing compared to standard diagnostic testing 
alone. Some even found that the diagnostic accuracy of certain pulmonary diseases was superior when it was assessed using f-US compared to a chest X-ray examination. ${ }^{18}$ One single centre study found a significant increase in diagnostic accuracy as well as in correctly prescribed treatment at 4 hours after the patient's arrival to the ED. This was made by adding an f-US of the heart, lungs and deep veins to standard clinical testing of patients with respiratory problems. ${ }^{3}$

Unfortunately, the results from these studies are not directly transferable to daily clinical practice as most of them are designed as single centre studies and some even performed by single investigators highly skilled in f-US. ${ }^{2} 31416$ 18-20 22

In this study, we aim to investigate whether adding an f-US of the lungs and heart to the standard diagnostic examination in patients with signs and symptoms of respiratory problems increases the proportion of patients with a correct diagnosis established at 4 hours after admittance. The study will be performed as a randomised pragmatic multicentre trial. The f-US will be performed by emergency physicians (EP) who attend to patients in the ED on a regular basis.

\section{METHODS \\ Design}

The trial is designed as a prospective randomised semiblinded, multicentre study with a parallel group design, an allocation ratio of 1:1 and as a superiority study. The protocol has been developed from the single centre study performed by one expert-investigator. ${ }^{3}$ As far as possible, we mimic the study design and transfer it into a multicentre design as well as comply with the few points of criticism the study has received. ${ }^{30}$

\section{Study flow and patient enrolment}

Patient enrolment started in October 2015 and is estimated to be finished in December 2016. We aim to include 288 patients admitted to nine different EDs. Enrolment will be performed in a non-consecutive manner by local investigators from each ED (figure 1). Patients comprise of those who are acutely admitted to the ED with symptoms of respiratory problems as their primary symptom. The investigator will screen the patients for inclusion according to the inclusion and exclusion criteria shortly after their arrival (box 1).

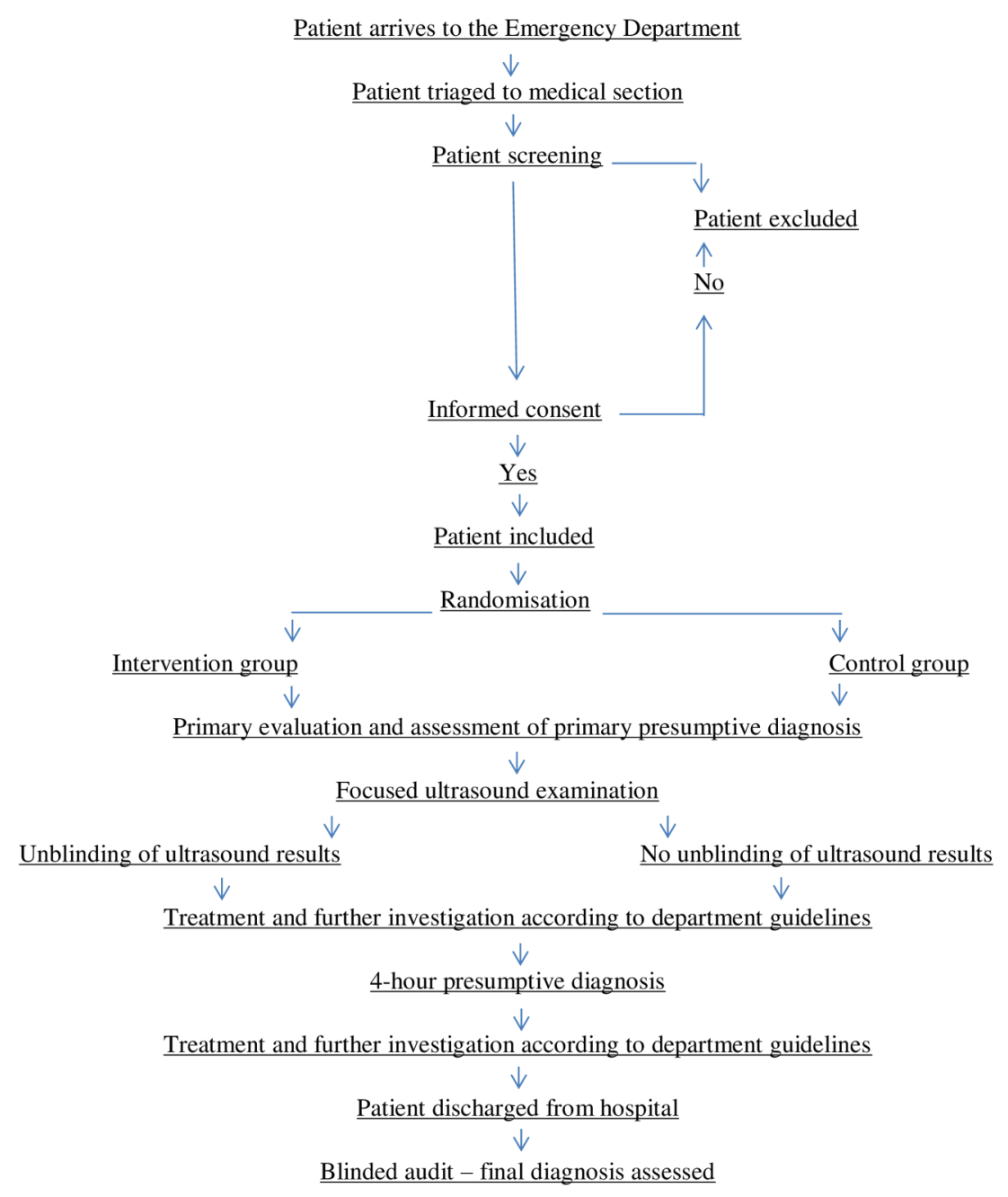

Figure 1 Enrolment and patient flow in the study. 


\section{Box 1 Inclusion and exclusion criteria for enrolment}

\section{Inclusion criteria.}

All four criteria have to be fulfilled.

1. The patient is 18 years or more.

2. The patient has arrived acutely to the emergency department (ED).

3. The patient presents with one or more of the following signs and symptoms as the primary symptom:

- Cough.

Dyspnoea.

- Chest pain.

- Respiration frequency $>20 / \mathrm{min}$.

- Peripheral oxygen saturation $<95 \%$.

4. Written informed consent from habile patient.

Exclusion criteria.

1. The patient is not capable of giving informed consent.

2. The focused ultrasound examination ( $f-U S$ ) of the lungs or the heart has already been performed by others than the investigator in relation to the primary assessment.

3. The randomisation and f-US cannot be performed within 4 hours from the patient's admittance to the ED.

Given that all inclusion criteria and none of the exclusion criteria are fulfilled, the investigator will provide the patient with oral and written information (see online supplementary appendix I) about the study in order to achieve informed consent whereafter he can draw a randomisation number in the assigned database.

A primary presumptive diagnosis upon the patient's arrival will be assessed by the EP for patients in both groups using the standard method of diagnostic assessment (eg, clinical assessment, blood samples, ECG, chest X-ray). Without knowledge of the primary presumptive diagnosis, the investigator performs an f-US of the heart and lungs on patients in the intervention and control group and gathers information on the basic clinical values upon the patient's arrival.

The f-US results from patients in the control group remain blinded for the treating physician. But for patients in the intervention group, the f-US results will be non-blinded to the treating physician once he has declared his primary presumptive diagnosis to the investigator. Non-blinding of f-US results will be performed by the investigator in oral and in writing, on a paper record, marked with the patient's personal ID number and stored in an accessible briefcase in the ED. Once the f-US results are non-blinded to the EP, he can re-evaluate his primary presumptive diagnosis and treatment.

Further diagnostic evaluation and treatment will proceed in accordance with the ED's clinical guidelines.

To ensure that we reach target sample size by an adequate patient enrolment, we have taken the following precautions: a pilot study was performed in the ED in one of the smallest hospitals participating in this trial and showed that an average of four eligible patients per day on an 8-hour shift in daytime. The investigators work between 1 and 4 days per week in the ED and they agreed on an inclusion rate of $20-25$ patients per investigator. This gives an estimate of 12-15 investigators necessary to include 288 patients and an estimate of inclusion time of $1-4$ weeks. To this we added the risk of investigators pausing inclusion due to busy shifts, holiday, sick leave or maternity leave or even dropping out of the project prior to completing their inclusion rates.

We here ended with a need for 20 investigators and an inclusion time of 15 months, including 3 months, for a local initiation of inclusion in the participating centres.

\section{Recruitment of investigators}

Investigators are found via MR's network of ultrasound enthusiasts and by oral presentations of the study on conferences and meetings among physicians and researchers of emergency medicine in Denmark. Once a potential investigator proved interest in participating in the study, MR supplied him or her with documents about the expected tasks as investigator, a description of the protocol, agreements on affiliation on the final product and a questionnaire regarding: their level of education, their attachment to the ED, their ultrasound skills, a description of the type of ultrasound equipment in the ED and the availability of this.

If the investigator fulfilled the basic requirements and still wished to become an investigator, MR contacted the management of the ED, by email, to ask for permission to initiate the trial in their department. Attached to this request was the study protocol along with the expected tasks of the investigator and the agreements on affiliation of the final product. Once the authorisation from the department leaders was attained, we agreed on a date for the educational programme, regarding the database and the ultrasound protocol. This programme was set to take place in the investigators' own environment. Until then, the investigators were provided with regular updates on study progress and with e-learn material about the ultrasound protocol.

\section{Standards and diagnoses}

As standard for the final diagnoses, we will make a blinded journal audit using the results from the f-US as well as predefined diagnostic criteria (see online supplementary appendix II). The audit will be undertaken once the patient has left the hospital. It will be performed by two physicians who, in case of discrepancy, will be complemented by a third auditor who will set the final decision.

As standard for a correct interpretation of the f-US, specialists in ultrasound of the lungs and the heart will perform a blinded audit using predefined diagnostic criteria (see online supplementary appendix III). The specialists will be blinded with respect to the investigator's evaluation of the f-US. All f-US recordings are saved as raw data.

The primary presumptive diagnosis is assessed by the EP who writes it on a predesigned paper record and 
hands it to the investigator prior to non-blinding of the f-US results. Subsequently, the investigator adds the primary presumptive diagnosis to the database.

The 4-hour presumptive diagnosis will be found by a blinded medical journal audit once the patient has left the hospital. For this, we use the last performed evaluation registered in the patient's medical record within 4 hours from admission to the ED. The specific cut-off at 4 hours is chosen because several EDs throughout Denmark have a 4-hour time limit when primary results (eg, blood sample, ECG, X-ray) and a presumptive diagnosis with relevant treatment should be started.

\section{Study sites}

The study sites comprise nine Danish EDs, hereof three EDs of tertiary referral hospitals. All participating EDs have admissions to the medical unit and represent a broad variation of the different constellations of EDs in Denmark concerning the number of admissions per year, opening hours as well as visitation of patients. Patients suspected of having a heart disease (eg, acute myocardial infarction or arrhythmia) are either admitted to the ED or directly to the cardiology department. Both constellations are represented in this study. There is no upper limit to the number of inclusions per ED. There are one to five investigators per ED and each investigator is expected to include 20-25 patients. List of study sites can be obtained from the corresponding author.

\section{Randomisation}

Randomisation is performed using an online block randomisation database (REDCap OPEN) in which permuted blocks of random numbers have been listed to ensure an equal number of patients in both study arms at each centre. ${ }^{31}$ The allocation sequence of randomisation numbers is generated by the data manager from REDCap OPEN and the project manager, MR, using an online random number service. ${ }^{32}$ The randomisation numbers are then transferred into the REDCap OPEN database assigned to this study. The investigator registers each patient in the database whereby he receives the patient's unique identification number and randomisation number which allocates the patient to either intervention or control group.

\section{Investigators and educational programme}

The local investigators are medical doctors who are either specialists or in specialist training and who receive patients in the ED on a regular basis. All the investigators use f-US in daily practice but have varying degrees of experience with f-US.

The project manager is responsible for educating the investigators in the data collection and the f-US protocol. This is undertaken as an e-learning presentation with instructional videos followed by a 4-hour onsite oral presentation of the project and the collection and registration of data as well as an individual evaluation of each investigator's f-US skills (eg, by hands on). ${ }^{33}$ All investigators must pass a multiple choice questionnaire test and be able to perform the f-US protocol prior to initiation of inclusion (see online supplementary appendix IV). During the period of inclusion, investigators can take daily contact to the project manager with any question. Further supervision will take place if necessary.

\section{The ultrasound protocol}

To reduce the time spent on f-US, but still keep the focus on the specific signs of pathology of the heart and lungs, which we look for in the acute situation, we have modified the ultrasound protocols as follows.

The f-US of the lungs is a modification of the ultrasound protocol used by Laursen et al. ${ }^{3}$ It is originally modified from the principles of lung ultrasound by Volpicelli $e t a t^{28}$ and Lichtenstein, ${ }^{34}$ and is performed as follows: the anterior and lateral part of thorax is divided into a superior and inferior quadrant. Each quadrant represents a zone in which the probe shall be placed centrally and create a transverse picture of the costae and pleura. A sequence of at minimum $4 \mathrm{~s}$, including at least one inspiration and one expiration, shall be recorded in all zones.

The focused cardiac ultrasound is performed according to the principles described in the international evidence-based guideline. ${ }^{9}$ The views used are the fourchamber picture of the heart achieved either from a subxiphoid or an apical window. A sequence of minimum $6 \mathrm{~s}$ of the heart shall be recorded.

\section{Blinding}

To ensure a proper blinded audit of the medical record, we have instructed investigators and treating EP to leave all information regarding the patient's unique identification number, randomisation number and f-US findings out of the medical record.

All f-US results from both groups are registered in the REDCap OPEN database only accessible for investigators and project managers. ${ }^{31}$ Only non-blinded f-US results are stored in an accessible briefcase in the ED for the EP to use.

\section{Outcomes}

\section{Primary outcome}

To investigate whether adding an f-US of the lungs and heart to the standard diagnostic examination in patients with signs and symptoms of respiratory problems increases the proportion of patients with a correct diagnosis established at 4 hours after admittance when f-US is performed by the local EP. The study will be performed as a randomised pragmatic multicentre trial.

\section{Secondary outcomes}

For the control and intervention group, we want to examine: 
1. The sensitivity, specificity, positive and negative predictive values and diagnostic accuracy of the primary presumptive diagnoses made upon arrival.

2. The proportion of patients receiving a correct primary presumptive diagnosis made upon arrival.

3. The proportion of $\mathrm{f}-\mathrm{US}$ with a correct diagnostic assessment.

4. The proportion of patients who within 4 hours after admission to the ED is given the correct treatment.

5. Time spent in the ED (hours).

6. Time spent at hospital (days).

7. The proportion of patients being discharged from the ED and directly to their home.

8. The proportion of patients being discharged from the ED to a hospital ward.

9. The proportion of patients being discharged from the ED to the intensive care unit.

10. The proportion of patients being readmitted to hospital within 30 days from discharge.

11. In-hospital mortality.

12. 30-Day mortality.

\section{Sample size}

The sample size estimate of this study is based on the results from the study by Laursen $e t a l^{3}$ We expect that $\sim 65 \%$ of the patients in the control group will have a correct presumptive diagnosis at 4 hours after admission to the ED. A clinically relevant absolute improvement of the tentative diagnosis using f-US in a multicentre study is set to be $15 \%$. To detect a $15 \%$ increase in the number of correct tentative diagnoses, from $65 \%$ in the control group to $80 \%$ in the intervention group, with an $80 \%$ chance for detection and a level of significance of 5\%, 272 included patients are needed. The estimated drop out is $6 \%$ and increases the number of inclusion to 288 patients. This gives 144 patients in each group (intervention/control group). Sample size calculations were made using the online database for clinical trials, Sealed Envelope. ${ }^{32}$

\section{Data analysis}

An intention-to-treat analysis will be performed on all participants and will serve as the primary analysis for all objectives in this study. In order to demonstrate a successful randomisation and the presence of even baseline groups, we register descriptive statistics (age, sex, smoking status, medical history, medication, triage colour) as well as clinical values and patient's primary symptom on admission at the ED. Data will be specified as follows: categorical data by number and proportion of patients. Continuous data by number of patients (n), mean, SD, median, minimum and maximum. Missing data will be handled by the use of the statistical method multiple imputation.

\section{Data analysis of primary end point}

To determine whether there is a difference in the proportion of patients with a correct/incorrect presumptive diagnosis between the intervention group and the control group, we will use a $\chi^{2}$ test or the Fischer exact test.

\section{Data analysis of secondary end points}

To compare the intervention group with the control group, we will use the following tests: for the comparison of means, the Student's t-test; for the comparison of medians, the Mann-Whitney test; and for the comparison of proportions, the $\chi^{2}$ or the Fisher exact test. A twosided significance level of $5 \%$ will be applied to all tests.

To assess the diagnostic accuracy of the primary presumptive diagnosis, the 4-hour presumptive diagnosis and the f-US, we will calculate the sensitivity, specificity, positive and negative predictive values and the diagnostic accuracy with 95\% CI based at a binominal distribution. To assess the interobserver agreement on the f-US results between investigators and the ultrasound specialists, we use the Cohen's $\kappa$ coefficient. Data analysis will be conducted using STATA Release V.14.0 (Stata Corp) including professional statistical advice.

\section{Data management}

Data specified in the study protocol will be directly documented and securely stored in the password protected database assigned for this study in REDCap OPEN, SDU. REDCap is a secure web application designed to support data capture for research at which the University of Southern Denmark has its own secure database access. ${ }^{35}$ The project manager is responsible for the database construction in cooperation with a data manager from OPEN (SDU) as well as for data acquisition, storage, monitoring flow and validation.

In this database, each patient receives a unique identification number securing patient identity. The investigators will only gain access to this database to withdraw a randomisation number and to enter data regarding the inclusion/exclusion, basic values upon arrival, f-US findings and the primary presumptive diagnoses.

Additional clinical data will be available from the electronic patient records in which there is no information on the randomisation number or f-US findings. Basic characteristics will be entered into the database by the project manager. Data regarding the blinded audit of the medical records will be entered into the database by the auditors who have no access to other data entry forms than those regarding the blinded audit. A data monitoring committee was not used. All the data reported are linked to each specific investigator and data reporting are monitored by project manager, MR, on a weekly basis.

F-US findings will be stored on secured external hard drives where patients will be entered by their unique identification number to secure patient identity and to enable a blinded audit by the specialists in f-US. Only the patient's informed consent for participation and non-blinded f-US findings are on paper format. No interim analysis or end point adjustments are planned. 
Box 2 Definition of adverse events leading to nonblinding of focused ultrasound examination results

For the purposes of this clinical trial, the definition of an adverse event/life-threatening condition will consist of any of the following:

- Pulmonary oedema. ${ }^{*}$

- Pneumothorax.

- Large pleural effusion. $\dagger$

- Pericardiac effusion.‡

- Heart failure.§

- Suspected pulmonary embolism.

- Aortic aneurysm/dissection.

* Identified as $\geq 3$ B-lines in two or more scanning zones anterior or lateral on each lung.

†Affecting breathing.

$\ddagger>0.5 \mathrm{~cm}$ measured in diastole.

$\S$ Not previously known and with ejection fraction $<45 \%$.

The final decision to end this trial before sample size is attained will be made by agreement between the project manager, MR, and her supervisors.

\section{ETHICS AND DISSEIMINATION}

The f-US is a method of investigation which has no known adverse effects. It will be performed in accordance with the local hospital guidelines. To avoid deprivation of a diagnostic tool in the control group, we have taken the following precaution for all included patients because f-US is gaining ground in few of the participating EDs and because the f-US findings are blinded in the control group: in case any f-US rises suspicion of a life-threatening condition, the f-US findings will immediately be nonblinded to the EP in charge of the patient (box 2).

The study will be conducted in accordance with the Helsinki II Declaration, ${ }^{36}$ and as approved by the Danish Data Protection Agency (identification number 13/ 12076), ${ }^{37}$ and the Committee on Biomedical Research Ethics for the Region of Southern Denmark (identification number S-20150090) with a dispensation from the normal rules for time for consideration since the f-US must be performed shortly after the patient has arrived to the ED. All data are stored and managed according to the laws and regulations of the Danish Data Protection Agency. The study is registered with Clinical trials (NCT02550184).

In the event that significant modifications to the protocol become necessary, MR will inform the coauthors, trial registers and investigators by mail and in person to ensure their implementation.

The results of this study will be published according to the CONSORT statement in peer-reviewed scientific journals regardless of the outcome. ${ }^{38}$

\section{STRENGTHS AND LIMITATIONS}

The generalisability of this study is supported by the multicentre design with investigators comprised of local EP from many specialties. The investigators represent the general level of expertise in f-US nationwide. The sample size is powered to detect a clinically significant improvement in the proportion of patients with a correct diagnosis and the blinded journal audit is strengthened by inclusion of f-US findings in the assessment of the final diagnosis.

The study has limitations: the investigators are recruited via MR's network of ultrasound enthusiasts. These doctors work in Danish EDs and they use ultrasound as part of their daily diagnostic procedures, but they cannot represent all doctors in the EDs. Furthermore, there is a risk of selection bias since patients are not consecutively enrolled. There is also a risk of non-blinding of f-US results before the primary or the 4-hour presumptive diagnosis has been attained since the investigators often hold the treatment responsibility. The study is not powered to investigate for differences in morbidity and mortality.

\section{CONCLUSION}

To our knowledge, this study is the first multicentre trial in the EDs to investigate whether an f-US in the hands of the EP can increase the proportion of patients with a correct diagnosis as well as the proportion of correctly initiated treatments on patients with signs of respiratory problems at 4 hours after their arrival to the ED. This trial holds the potential to develop evidence-based optimisation of early diagnostic accuracy in these patients.

\section{Author affiliations}

${ }^{1}$ Department of Surgery (A), Odense University Hospital, Svendborg, Denmark ${ }^{2}$ Institute of Clinical Research, University of Southern Denmark, SDU-Odense, Denmark

${ }^{3}$ Department of Internal Medicine \& Emergency Medicine (M/FAM), Odense University Hospital, Svendborg, Denmark

${ }^{4}$ Department of Respiratory Medicine, Odense University Hospital, Odense, Denmark

${ }^{5}$ Department of Emergency Medicine, Odense University Hospital, Odense, Denmark

Acknowledgements MR would like to thank associate professor M Brabrand, MD, PhD and H Sheta, MD for their time spent on critical and professional inputs during the idea development of this study.

Contributors $\mathrm{CBL}, \mathrm{ATL}, \mathrm{GB}$ and MR contributed to the conception and design of the study. MR and CBL drafted the protocol and MR then wrote the protocol in accordance to the coauthors' contributions. All authors approved the final draft of the protocol. MR is the project manager and in charge of all aspects of database management, education of investigators and conduction of inclusion. All authors contributed to the writing and the reviewing of this article.

Funding This trial is supported partly by the Department of Medicine/The Emergency Department and the Department of Surgery $(A)$ at Odense University Hospital-Svendborg and partly by grants from the University of Southern Denmark, the PhD foundation at the Region of Southern Denmark and the Institute of Regional Research at Odense University Hospital-Svendborg. ATL is supported by an unrestricted grant from the philanthropic fund, the Tryg Foundation, given to the University of Southern Denmark.

Competing interests MR has been paid as instructor for courses in ultrasound organised by USabcd. CBL has received royalties from USabcd as author of e-learn material. 
Ethics approval Approved by the Danish Data Protection Agency and the Regional Committees on Health Research Ethics for Southern Denmark.

Provenance and peer review Not commissioned; externally peer reviewed.

Open Access This is an Open Access article distributed in accordance with the Creative Commons Attribution Non Commercial (CC BY-NC 4.0) license, which permits others to distribute, remix, adapt, build upon this work noncommercially, and license their derivative works on different terms, provided the original work is properly cited and the use is non-commercial. See: http:// creativecommons.org/licenses/by-nc/4.0/

\section{REFERENCES}

1. Ray $P$, Birolleau $S$, Lefort $Y$, et al. Acute respiratory failure in the elderly: etiology, emergency diagnosis and prognosis. Crit Care 2006; 10:R82.

2. Laursen $\mathrm{CB}$, Sloth $\mathrm{E}$, Lambrechtsen J, et al. Focused sonography of the heart, lungs, and deep veins identifies missed life-threatening conditions in admitted patients with acute respiratory symptoms. Chest 2013;144:1868-75.

3. Laursen CB, Sloth E, Lassen AT, et al. Point-of-care ultrasonography in patients admitted with respiratory symptoms: a single-blind, randomised controlled trial. Lancet Respir Med 2014;2:638-46.

4. Peacock WF, Braunwald E, Abraham W, et al. National Heart, Lung, and Blood Institute working group on emergency department management of acute heart failure: research challenges and opportunities. J Am Coll Cardiol 2010;56:343-51.

5. Wang CS, FitzGerald JM, Schulzer M, et al. Does this dyspneic patient in the emergency department have congestive heart failure? JAMA 2005;294:1944-56.

6. Delerme S, Ray P. Acute respiratory failure in the elderly: diagnosis and prognosis. Age Ageing 2008;37:251-7.

7. Spencer KT, Kimura BJ, Korcarz CE, et al. Focused cardiac ultrasound: recommendations from the American Society of Echocardiography. J Am Soc Echocardiogr 2013;26:567-81.

8. Volpicelli G, Elbarbary M, Blaivas M, et al. International evidence-based recommendations for point-of-care lung ultrasound. Intensive Care Med 2012;38:577-91.

9. Via G, Hussain A, Wells M, et al. International evidence-based recommendations for focused cardiac ultrasound. J Am Soc Echocardiogr 2014;27:683.e1-83.e33.

10. American College of Emergency Physicians. Emergency ultrasound guidelines. Ann Emerg Med 2009;53:550-70.

11. Laursen CB, Nielsen K, Riishede M, et al. A framework for implementation, education, research and clinical use of ultrasound in emergency departments by the Danish Society for Emergency Medicine. Scand J Trauma Resusc Emerg Med 2014;22:25.

12. Perera $P$, Mailhot $T$, Riley $D$, et al. The RUSH examination: rapid ultrasound in SHock in the evaluation of the critically ill. Emerg Med Clin North Am 2010;28:29-56, vii.

13. Manno E, Navarra M, Faccio L, et al. Deep impact of ultrasound in the intensive care unit: the "ICU-sound" protocol. Anesthesiology 2012;117:801-9.

14. Pivetta E, Goffi A, Lupia E, et al. Lung ultrasound-implemented diagnosis of acute decompensated heart failure in the ED: a SIMEU multicenter study. Chest 2015;148:202-10.

15. Willenheimer RB, Israelsson BA, Cline CM, et al. Simplified echocardiography in the diagnosis of heart failure. Scand Cardiovasc J 1997;31:9-16.

16. Zanobetti M, Poggioni C, Pini R. Can chest ultrasonography replace standard chest radiography for evaluation of acute dyspnea in the ED? Chest 2011;139:1140-7.

17. Lichtenstein DA, Mezière GA. Relevance of lung ultrasound in the diagnosis of acute respiratory failure: the BLUE protocol. Chest 2008;134:117-25.
18. Xirouchaki N, Magkanas E, Vaporidi K, et al. Lung ultrasound in critically ill patients: comparison with bedside chest radiography. Intensive Care Med 2011;37:1488-93.

19. Dexheimer Neto FL, Andrade JM, Raupp AC, et al. Diagnostic accuracy of the bedside lung ultrasound in emergency protocol for the diagnosis of acute respiratory failure in spontaneously breathing patients. J Bras Pneumol 2015;41:58-64.

20. Russell FM, Ehrman RR, Cosby K, et al. Diagnosing acute heart failure in patients with undifferentiated dyspnea: a lung and cardiac ultrasound (LuCUS) protocol. Acad Emerg Med 2015;22:182-91.

21. Peris A, Tutino L, Zagli G, et al. The use of point-of-care bedside lung ultrasound significantly reduces the number of radiographs and computed tomography scans in critically ill patients. Anesth Analg 2010;111:687-92.

22. Gallard E, Redonnet JP, Bourcier JE, et al. Diagnostic performance of cardiopulmonary ultrasound performed by the emergency physician in the management of acute dyspnea. Am J Emerg Med 2015;33:352-8.

23. Prosen G, Klemen P, Štrnad M, et al. Combination of lung ultrasound (a comet-tail sign) and $\mathrm{N}$-terminal pro-brain natriuretic peptide in differentiating acute heart failure from chronic obstructive pulmonary disease and asthma as cause of acute dyspnea in prehospital emergency setting. Crit Care 2011;15:R114.

24. Xirouchaki N, Kondili E, Prinianakis G, et al. Impact of lung ultrasound on clinical decision-making in critically ill patients. Intensive Care Med 2014;40:57-65.

25. Goffi A, Pivetta E, Lupia E, et al. Has lung ultrasound an impact on the management of patients with acute dyspnea in the emergency department? Crit Care 2013;17:R180.

26. Parlamento S, Copetti R, Di Bartolomeo S. Evaluation of lung ultrasound for the diagnosis of pneumonia in the ED. Am J Emerg Med 2009;27:379-84.

27. Volpicelli G. Sonographic diagnosis of pneumothorax. Intensive Care Med 2011;37:224-32

28. Volpicelli G, Mussa A, Garofalo G, et al. Bedside lung ultrasound in the assessment of alveolar-interstitial syndrome. Am J Emerg Med 2006;24:689-96.

29. Arntfield RT, Millington SJ. Point of care cardiac ultrasound applications in the emergency department and intensive care unit-a review. Curr Cardiol Rev 2012;8:98-108.

30. Ramirez P, Torres A. Should ultrasound be included in the initial assessment of respiratory patients? Lancet Respir Med 2014;2:599-600.

31. REDCap-Research Electronic Data Capture. http://project-redcap. org/ (assessed 3 Dec 2015)

32. Sealed envelope. https://www.sealedenvelope.com/power/ binary-superiority/ (assessed 14 Apr 2015).

33. Foss KT, Subhi Y, Aagaard R, et al. Developing an emergency ultrasound app-a collaborative project between clinicians from different universities. Scand J Trauma Resusc Emerg Med 2015;23:47.

34. Lichtenstein D. General ultrasound in the critically ill. Berlin, Heidelberg, Germany: Springer-Verlag, 2007.

35. REDCap OPEN (Research Electronic Data Capture, Odense Patient data Explorative Network), Department of Clinical Research, University of Southern Denmark (assessed 3 Dec 2015). https://open.rsyd.dk/redcap/

36. WMA Declaration of Helsinki-Ethical Principles for Medical Research Involving Human Subjects. http://www.wma.net/en/ 30publications/10policies/b3/ (assessed $30 \mathrm{Apr} 2015$ ).

37. Datatilsynet. The Act on Processing Personal Data. http://www. datatilsynet.dk/english/the-act-on-processing-of-personal-data/ read-the-act-on-processing-of-personal-data/ compiled-version-of-the-act-on-processing-of-personal-data/ (assessed 26 Nov 2015).

38. The Consort 2010 guideline (CONsolidated Standards of Reporting Trials). http://www.consort-statement.org/media/default/downloads/ CONSORT\%202010\%20Checklist.pdf (assessed 3 Dec 2015). 